\title{
Temporal Bone and Cerebellopontine Angle Epidermoid Resulting in Facial Nerve Paralysis: Resection and Facial Nerve Coaptation
}

\author{
Alexander G. Bien ${ }^{1,2}$ Christine S. Kim² Tyler J. Kenning ${ }^{3}$
}

${ }^{1}$ Albany Ear, Nose, and Throat Services, Albany, New York, United States

Address for correspondence Alexander G. Bien, MD, FACS, Albany

2 Division of Otolaryngology, Albany Medical Center, Albany, New York, ENT \& Allergy Services, PC., 400 Patroon Creek Blvd, Ste 205, Albany, United States

${ }^{3}$ Department of Neurosurgery, Albany Medical Center, Albany, NY 12206 (e-mail: bienag@gmail.com).

New York, United States

J Neurol Surg B 2019;80(suppl S3):S314-S315.

\begin{abstract}
Objectives Demonstrate the utilization of a transcochlear approach for resection of an epidermoid involving the temporal bone and cerebellopontine angle (CPA) with endto-end facial nerve coaptation.

Designs Single case-based operative video.

Setting Tertiary center with dedicated skull base team.

Participants The patient is a 50-year-old left handed male with a history of a remote left Bell's palsy, left sudden sensorineural hearing loss, and a rapidly progressive facial nerve paralysis. His balance was impaired, and his videonystagmography showed a significant left sided peripheral vestibular weakness. Computed tomography (CT) scan showed an erosive lesion of his left temporal bone involving the cochlea and semicircular canals, and magnetic resonance imaging (MRI) showed a T2 hyperintense lesion with restricted diffusion and no enhancement on postcontrast $\mathrm{T} 1$ sequences.

Main Outcome Measures Gross total resection of the epidermoid, recovery of facial

\section{Keywords}

- epidermoid tumor

- transcochlear craniotomy

- facial nerve paralysis

- nerve coaptation

- temporal bone

- cerebellopontine angle

- facial nerve anastamosis nerve function, balance improvement.

Results The patient underwent resection via a transcochlear approach. The tumor involved the epitympanum and eroded the semicircular canals, vestibule, and basal turn of the cochlea. Gross total tumor resection was attained. The facial nerve was isolated in the mastoid and tympanic segments, traced proximally to the geniculate ganglion, and then into the internal auditory canal (IAC). The nerve was discontinuous in the distal IAC and a reactive neuroma was resected. The facial nerve was mobilized and an end-to-end coaptation was performed in the CPA using a collagen tubule. The 3-month postoperative MRI showed no residual or recurrent disease. His postoperative balance was improved. Partial facial nerve recovery is not expected prior to 9 to 12 months.

The link to the video can be found at: https://youtu.be/C6N8qPwBt2Y.
\end{abstract}

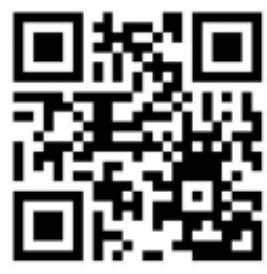

received

May 30, 2018

accepted

August 19, 2018

published online

October 31, 2018

www.thieme.com/skullbasevideos

www.thieme.com/jnlsbvideos

DOI https://doi.org/

10.1055/s-0038-1673704.

ISSN 2193-6331. (c) 2019 Georg Thieme Verlag KG

Stuttgart · New York
License terms

(c) (i) $\ominus$ (\$) 


\section{Conflict of Interest}

None.

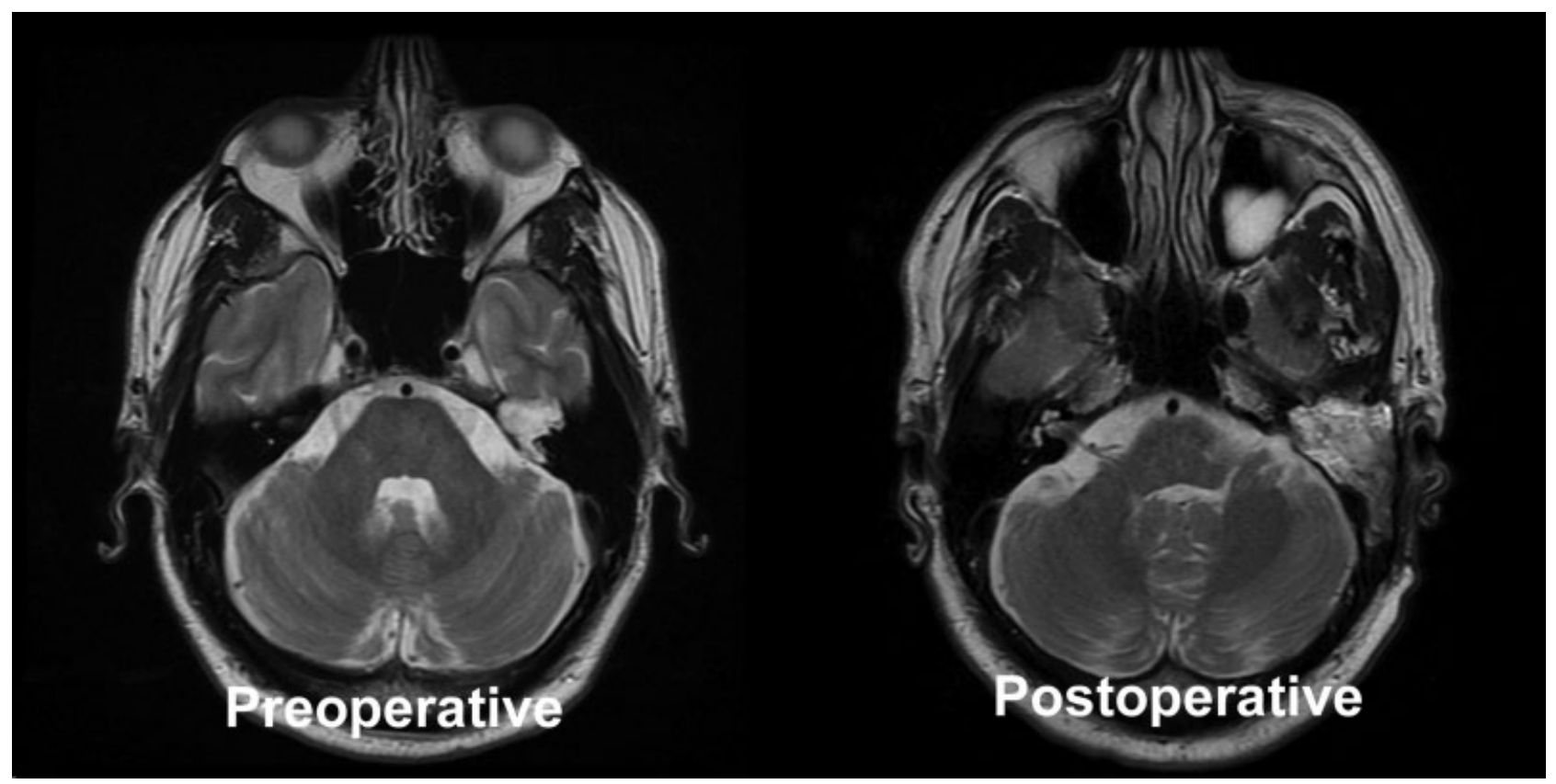

Fig. 1 Preoperative and postoperative axial T2 magnetic resonance imaging sequences showing complete resection of a left temporal bone and cerebellopontine angle epidermoid tumor.

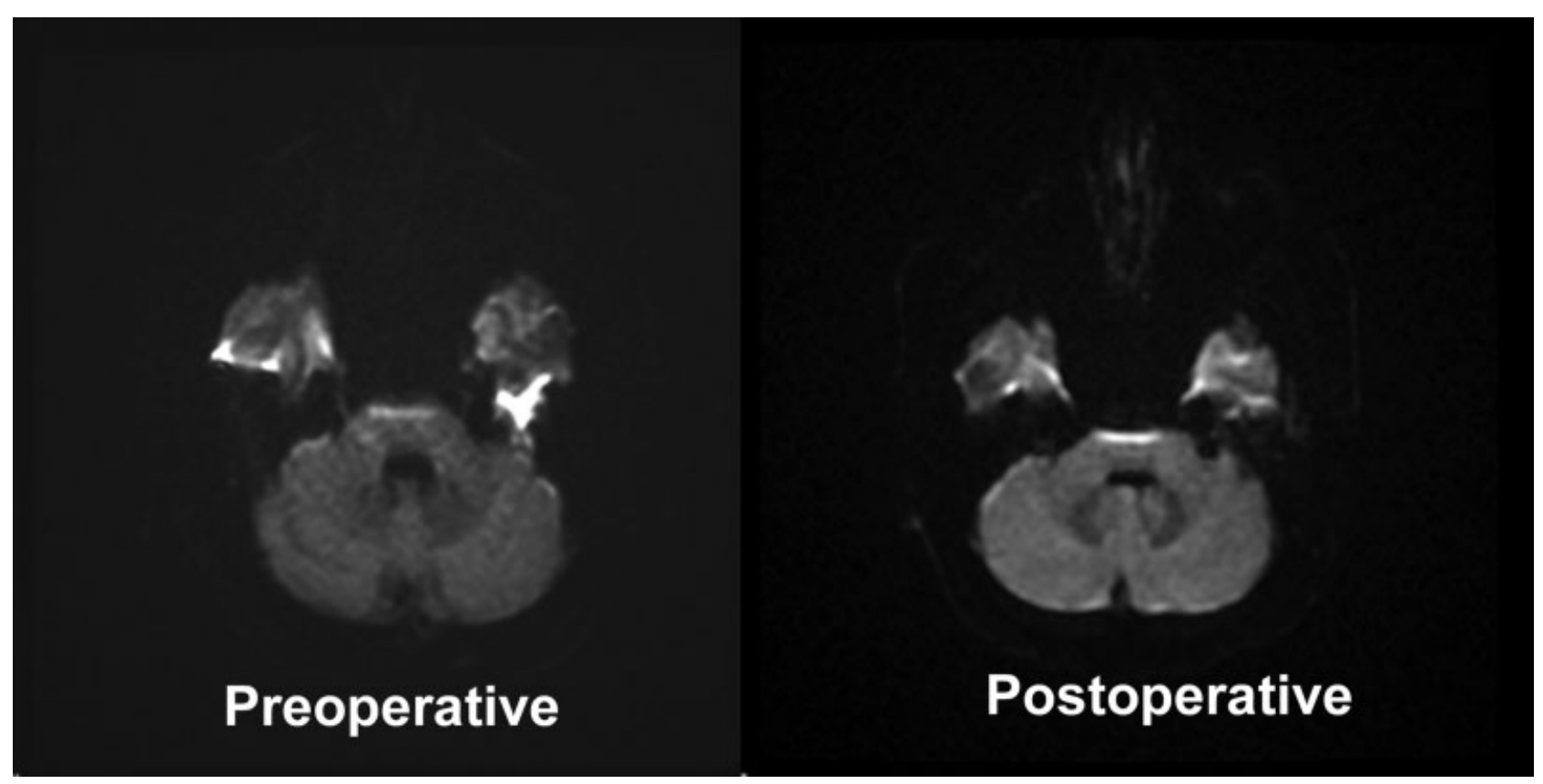

Fig. 2 Preoperative and postoperative axial diffusion-weighted magnetic resonance imaging sequences showing complete resection of a left temporal bone and cerebellopontine angle epidermoid tumor. 